\title{
CNC Machines Monitoring Using Sensorial Stochastic Models
}

\author{
Manuel M. Barata, Ricardo R. Jardim Gonçalves, Adolfo S. Steiger \\ Garção, "José Álvaro Assis Lopes \\ Universidade Nova de Lisboa - FCT \\ Departamento deEngenharia Electrotécnica \\ Quinta da Torre, 2825 Monte de Caparica \\ Portugal \\ E-Mailmmb@uninova.pt \\ *Instituto Superior Técnico, Secção Autónoma de Economia e Gestão
}

\begin{abstract}
This paper presents the work that is being done by the Intelligent Robotics Group on a CNC Monitoring and Prognosis System. It is based on an integrated hardware/software environment including $\mathrm{CNC}$ lathe and mill machines. The machining process is monitored in real time using sensors for vibrations, sound and power consumption. This paper presents our approach for sensor signal modelling using auto regressive stochastic models. Models for machining process characterisation have already been investigated for different situations. The applicability of the $\operatorname{ARMA}(p, q)$ model for process monitoring and evolution tracking, are discussed as well as the results obtained with real data are presented.
\end{abstract}

Keywords

Monitoring, Prognosis, CNC, Sensor Integration, Expert Supervision, Stochastic Process.

\section{INTRODUCTION}

CNC machines play an important role in Flexible Manufacturing Systems: They produce parts to be assembled. Solving the problem of making products featuring the ability of defects forecasting will considerably contribute to improve the system's productivity and product quality. This goal leads to an exhaustive analysis of related tasks, in order to identify and enumerate the relevant aspects suitable to be improved. In our case numerically controlled machines work as a test bed for experimentation and demonstration.

System's degradation, opposite to random accidents, has to be adequately monitored and evaluated if we are committed to corrective action or intervention. As almost in all the defective situations, a broad band of possibilities can occur: from well identified symptoms to very fuzzy 
and unclear scenarios. Dynamics in those cases will play a major role, because decision will be based not only on statistical measured values, but also on their temporal evolution. Advanced monitoring systems, depending on the particular design ambition, can address or not, automatic refinement of decision making mechanisms. That stresses the fact that statistical accumulation and assessment are important functions to be incorporated in that kind of systems.

Faults can occur randomly or as a consequence of structural internal degradation. Little hope exists for the first case. However, the second one opens the field for prediction of deviation of quality, enlarging the state-of-the-art methodologies. Prediction of faults can represent an important economic issue as an effective technique aimed to operate in run time and not based on a posterior analysis of samples.

An experimental setup specially developed by our group for experimental research on monitoring the machining process was developed and it has been used for the assessment of our developed models. Additional information describing the system's architecture and processing facilities are described in (Steiger-Garção 1989), (Barata 1990a), (Barata 1990b) and (Barata 1990c). In this paper we present the work done in sensorial data characterisation using autoregressive stochastic process and, in our point of view, is a refinement of the approach presented in (Barata 1994) because it contributes with an additional technique for the machining process monitoring and failure prognosis.

\section{APPROACH STRATEGY}

\subsection{Machining process characterisation}

Monitoring and Supervisioned action based on sensors is considered. Sensing, characterisation of the different participating processes and learning their behaviour, are the base for the implementation of decision making mechanisms. Our models are mainly supported by learning. They are built taking into consideration the process physics, and are tuned and trained under real time sensor data acquisition.

To build a suitable model of the machining process we need to identify a set of low level building blocks (process steps). These blocks are commonly called geometric machining features. The machining of a specific piece is thus understood as the execution, in a proper order, of a sequence of these blocks. The corresponding Numerical Control (NC) programs can be understood as a way of implementation of such building blocks. Inspecting several designs of different pieces and the corresponding NC programs, a great level of similarity between the segments of NC instructions corresponding to the machining of the same geometric feature in different pieces can be found. This particularity and considering that the machine's behaviour is mainly determined by the NC instructions it executes, it is plausible to expect a characterisation of the machining process based on machining features, independently of a specific piece. This characterisation is done by learning the associated sensorial patterns in terms of good and bad situations or when this is not possible in terms of the good situations already found. This approach was previously presented in (Barata 94) and we shortly describe it here in order to give the reader a better understanding of the problem we are addressing.

\subsection{Machining process model}

At physical level the machine, machining process and installed sensors can be modelled as a system having one input ( the sequence of the NC instructions) and several outputs (one for each sensor). Such a model is presented in figure 1. During the machining process, while executing the $\mathrm{NC}$ instructions, each installed sensor presents a signal that can be used as a signature for the 
characterisation of the process. For each status (execution of one NC instruction) the installed sensors output a proper signal pattern. We understand such patterns as the system response to the given stimulus. In part b) of figure 1 it is sketched how the sensor data can be related to the $\mathrm{CN}$ commands during the execution of a complete NC program. Thus the time series of the data must be labelled with the $\mathrm{CN}$ command that was in execution during data capture.

According to figure 1 the machining process is characterised by the $\mathrm{NC}$ instruction and the sensor patterns produced by the sensors while the NC instruction is being executed. Accordingly, the first step is to get knowledge about the sensors' behaviour for the set of all situations. Using Pattern recognition terminology, we denote by class the execution of each $\mathrm{NC}$ instruction, and the sensorial signal characterisation by features.

After getting knowledge about the sensor data behaviour, the sensor model can be implemented. That model includes the representation of the nature of the low level data processing and the feature extraction method. Also the correlation of the resulting features and the process status related to the collected data must be evaluated. After this evaluation decisions can be made about which features are good or not for process characterisation. That leads us to the selection of a correct feature extraction method. The reader can find more detailed information about this subject on (Barata 1994). In (Rauber 1993) a tool developed for statistical characteristics evaluation of the feature vector is presented. For, example using the FFT for low level processing, it can be determined which subset of harmonics represents the best correlation with the variable class values (Guinea 1991). Some results of applying this procedure are presented in (Barata 1992), (Rauber 1993), (Barata 1994).

a)
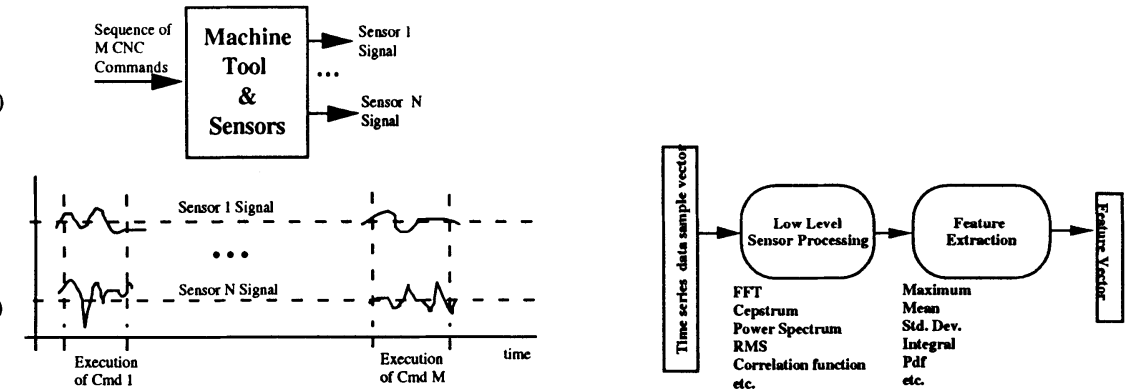

Figure 1 Machine model and sensor data capturing.

Figure 2 P.R. feature extraction.

Figure 2 summarises the block diagram of low level sensor data processing and feature extraction. Besides the mentioned techniques, the work presented in this paper concentrates in the application of the theory of Stochastic Time Series Analysis for doing low level sensor processing and feature extraction. This approach is motivated by the nature of the data produced by some kind of sensors that present some stationary random characteristics, e.g. the sound or vibrations of a cutting process. For several repetitions of the same machining process, these sensors never reproduce the same signal pattern but similar patterns evidencing some randomness.

\section{DISCRETE SYSTEMS MODELLING USING TRANSFER FUNCTION}

\subsection{Discrete systems}

A discrete system is a rule for assigning to a sequence $f[n]$ another sequence $g[n]$. Thus, a discrete system is a mapping (transformation) of the sequence $f[n]$ into the sequence $g[n]$. We 
shall use the notation $g[n]=L\{f[n]\}$ for this mapping. The sequence $f[n]$ will be called de input, and the sequence $g[n]$ the output, or response (Papoulis 1984).

The response of a discrete system to the delta sequence is usually called the system transfer function:

$h[n]=L\{\delta[n]\}$.

and it defines the response of the system to an input sequence $f[n]$. It is possible to determine the response of the system doing the discrete convolution between the input sequence $f[n]$ and the system function $h[n]$ :

$g[n]=f[n] * h[n]=\sum_{k=-\infty}^{\infty} f[k] h[n-k]=\sum_{k=-\infty}^{\infty} f[n-k] h[k]$.

For our aims, the system transfer function must have the property of linearity, time invariance and causality given respectively by the following equations:

$$
\begin{aligned}
& L\left\{a_{1} f_{1}[n]+a_{2} f_{2}[n]\right\}=L\left\{a_{1} f_{1}[n]\right\}+L\left\{a_{2} f_{2}[n]\right\} \text { for any } a_{1}, a_{2}, f_{1}[n] \text { and } f_{2}[n] . \\
& L\{f[n-k]\}=g[n-k] \text { and } h[n]=0 \text { for } n<0 .
\end{aligned}
$$

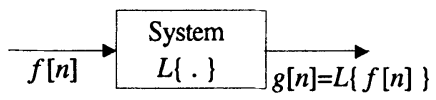

Figure 3 Discrete system model.

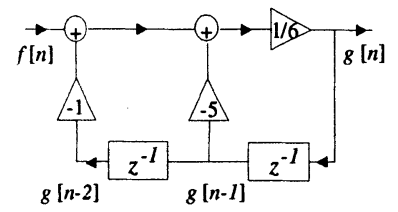

Figure 4 Discrete realisation of a system.

\subsection{System Function}

Computing the $\mathrm{Z}$ transform of the system transfer function $h[n]$, we obtain the usually called System Function in the complex $Z$ domain:

$H(z)=\sum_{n=-\infty}^{\infty} h[n] z^{-n} ; L\left\{z^{n}\right\}=H(z) z^{n}$.

in words: If the input sequence is $z^{n}$ the corresponding output is the same sequence multiplied by the System Function $H(z)$. Discrete systems are useful for simulating analogue systems in a computer. For example if we program the system $6 g[n]+5 g[n-1]+g[n-2]=f[n]$ the corresponding system function $H(z)$ is :

$H(z)=\frac{1}{6+5 z^{-1}+z^{-2}}$

The block diagram of the corresponding filter is presented in figure 4 . The $z^{-1}$ boxes correspond to one sample time delay and the triangles are amplifiers.

\section{AUTOREGRESSIVE MODELS}

Sensorial data can be modelled using the statistical models and techniques developed for characterisation of stochastic time series. They assume that most of the stochastic process 
realisations can be modelled by filtering white noise. This approach was first presented by (Box.Jenkins 1970). In this sense white noise is a special stochastic process, which realisations' $\varepsilon[n]$ form the input of a system and the corresponding response $g[n]$ is another stochastic process realisation. These two stochastic process, and of course the corresponding realisations, are related by the system transfer function $H(z)$. The white noise process is stationary and has: constant expected value $E\{\varepsilon[n]\}=\eta_{\varepsilon}$, normally $\eta_{\varepsilon}=0$, constant variance $V\{\varepsilon[n]\}=\sigma_{\varepsilon}^{2}$ and correlation:

$\rho_{k}=\left\{\begin{array}{l}1 \text { for } k=0 \\ 0 \text { for } k \neq 0\end{array}\right.$

In general if the system input sequence $f[n]$ has the autocorrelation function $\phi_{f f}(n)=E\{f[k] f[k+n]\}$ the correlation between the input and output is:

$\phi_{g f}(n)=E\{g[k] f[k+n]\}=E\left\{g[k-n] f[k]=\phi_{f_{g}}(-n)\right.$

If $\Phi_{g f}(z)$ and $\Phi_{f f}(z)$ are the $\mathrm{Z}$ transforms of $\phi_{g f}(n)$ and $\phi_{f f}(n)$ the following relation holds:

$\Phi_{g f}(z)=H(z) \Phi_{f f}(z)$

It describes the effect of the system transfer function on the input sequence. Detailed presentation of this subject can be found in (Box-Jenkins 1970), (Abraham 1983), (Harvey 1992).

\section{1 $\operatorname{ARMA}(p, q)$ Models}

A stochastic process $g[n]$ is $\operatorname{ARMA}(p, q)$ when it satisfies the following difference stochastic equation:

$g[n]-\phi_{1} g[n-1]-\cdots-\phi_{p} g[n-p]=\varepsilon[n]-\theta_{1} \varepsilon[n-1]-\cdots-\theta_{q} \varepsilon[n-q]$

and the corresponding system transfer function is:

$H(z)=\frac{1-\theta_{1} z^{-1}-\cdots-\theta_{q} z^{-q}}{1-\phi_{1} z^{-1}-\cdots \phi_{p} z^{-p}}$

For sensorial data modelling, given a sensor times series sample $g[n]$, the goal is to find a system transfer function, such that when feed by white noise results in a similar process realisation. It is necessary to determine the values of $p$ and $q$, and the coefficient values $\phi_{1} L \phi_{p}$ and $\theta_{1} L \theta_{q}$. This process is iterative and can be done using a statistical package, e.g. SPSS (SPSS 1993).

\section{APPLICATION TO MONITORING THE MACHINING PROCESS}

Stochastic autoregressive models $\operatorname{ARMA}(p, q)$ described in paragraph four can be used for monitoring the machining process in three ways:

- Doing low level sensor signal processing and feature extraction. Using the sensor data, the parameters of the $\operatorname{ARMA}(p, q)$ model are estimated. The feature vector corresponds to the vector built with the $p, q$ parameters estimated. In this case the feature vector describes the transfer function of a filter that transforms white noise in a signal similar to the sensor output. The clustering of the vector of $p, q$ parameters is used by the upper level for identifying the class of the signal, i.e. identify the status of the process. 
- As identifiers of the sensor data signal. In this situation they work as signal predictors, and match the signal to the class model that presents the lowest error in prediction. A monitor can decide about the situation by comparing the identified class with the expected one.

- Tracking the evolution of some situation, e.g. the lubrication status. In this case the $\operatorname{ARMA}(p, q)$ model is used for doing continuous parameter estimation and the feature vector is used for tracking a typical known path. If critical situations are marked in the path, it is possible to predict how far is the current vector from that zone. In this case tracking the parameters is equivalent to track the system's transfer function. If the transfer function is changing that means a change in the system.

Figure 5 a) presents the block diagram corresponding to the low level sensor processing and feature extraction. An identifier uses the feature vector for identifying the class (i.e. process status) by comparing it with a set of feature clusters learned during a training phase.

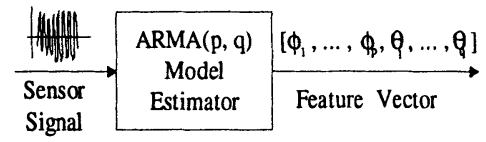

a)

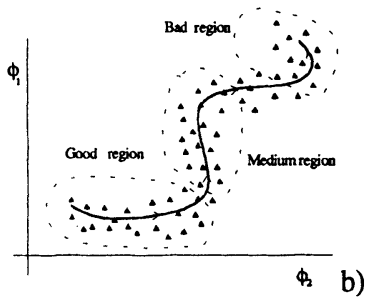

\$ b)

Figure 5 a) Application of the $A R M A(p, q)$ model for low level signal processing and b) Application of $\operatorname{ARMA}(2,0)$ for process quality tracking.

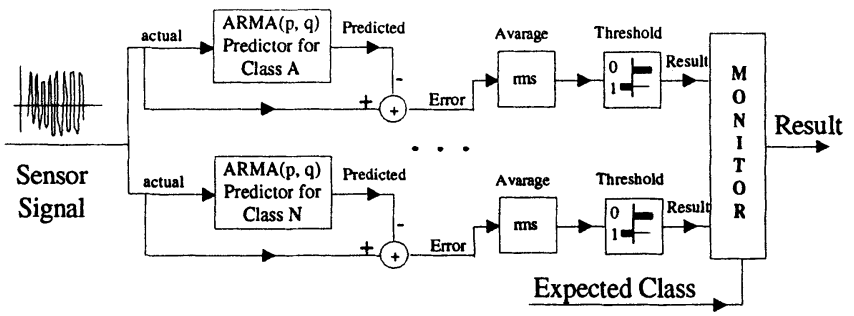

Figure 6 Application of the $\operatorname{ARMA}(p, q)$ model for signal class identification

The second case is illustrated in figure 6. Here we have a fixed $\operatorname{ARMA}(p, q)$ model for each class of the process status. Each model works in parallel as a signal predictor, and compares the error between the predicted and incoming signal. For a given situation the model presenting the lowest error level is selected for signal identification. Each threshold unit is programmed with a threshold value computed during the estimation of the corresponding class model. The monitor decides about the situation by comparing the expected class value with the class identified. The average units are necessary, because during estimation, the error estimated is based on the average prediction error. Moreover it does some filtration avoiding false errors due to noise.

An example of parameter tracking is illustrated in figure $5 \mathrm{~b}$ ). In this case, for simplicity of explanation, it was assumed a model with two parameters (higher dimensions are easily extrapolated). The construction of the parameters' path is done by a previous correlation with the model's parameters and known situations. For instance, suppose that the example of figure $5 \mathrm{~b}$ ) explains the lubrication status of some component. To have that characterisation, it was necessary to do a first track of the process during a complete phase, starting with a good level of 
lubrication, passing by intermediate levels until a low level was reached. By this way we have learned the process characterisation and recorded the parameters' clustering for all situations.

\section{EXPERIMENTS DONE}

In order to evaluate the applicability of the monitoring approaches presented in the previous paragraph, it was decided to do preliminary experiments. Four sensors for machining process sensing were installed in a lathe STARTURN 4. This machine is a small laboratorial CNC lathe with $1 / 2 \mathrm{Hp}$ spindle power and the axes are driven by step motors. One sensor, senses the mains current consumed by the machine. Besides some constant consumption done by the machine's electronics, the main consumption is related with the spindle motor. A second sensor is a microphone that captures the machine working sound. The third and four are two accelerometers installed on the tool handler carriage. One accelerometer detects the vibrations on the $\mathrm{X}$ axis direction and the other for the $\mathrm{Z}$ axis. Figure 7 illustrates the installation of the sensors on the machine.

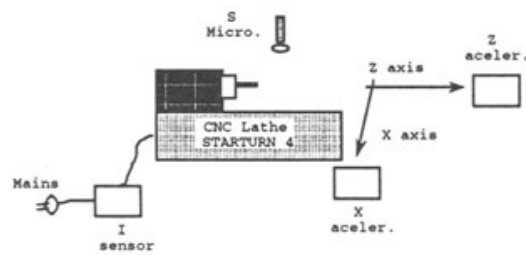

Figure 7 Locations of lathe sensors.
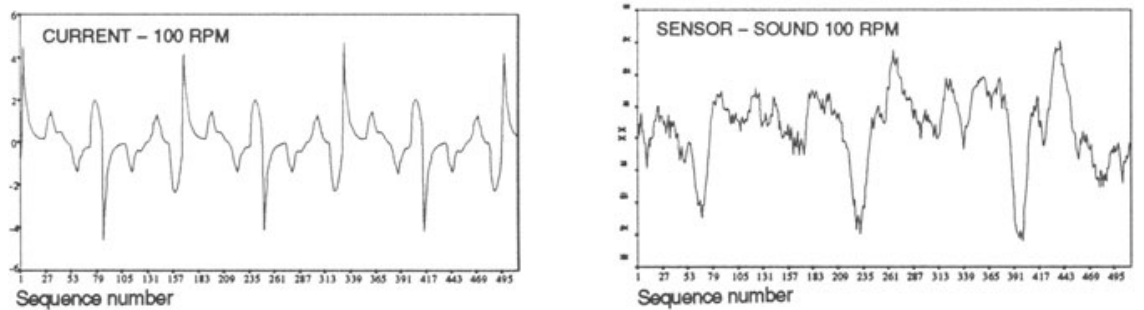

Figure 8 Plotting of current and sound sensors' data collected with spindle at 100 RPM.
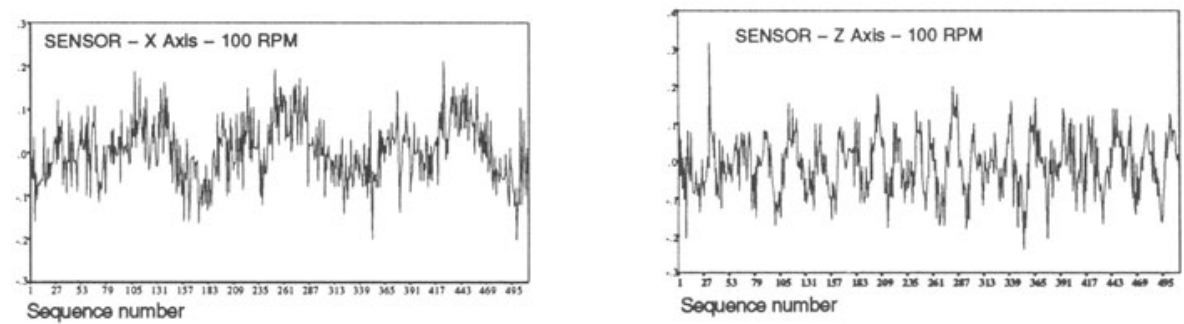

Figure 9 Plotting of axis $X$ and $Z$ sensors' data collected with spindle at 100R RPM.

The experiments performed correspond to a small subset of all machining situations. It was decided to analyse only the possibility of identification of the spindle speed by means of the 
described sensors. Sensor data was captured for the following spindle rotations: 100RPM, 300RPM, 500RPM, 700RPM and 900RPM. The sampling rate was $8192 \mathrm{~Hz}$, corresponding to $122,07 \mu \mathrm{s}$ sampling intervals.

Figures 8 and 9 depict the series got from sensors while the spindle was operated at 100 RPM.

In order to obtain the correspondent $\operatorname{ARMA}(p, q)$ models, blocks of 512 data sample points were considered. The procedure described below for determining the $\operatorname{ARMA}(p, q)$ model using the axis $\mathrm{Z}$ sensor data was performed similarly for the data of the other sensors.

There is a model building procedure proposed by (Box-Jenkins 1970). This procedure was followed, and consists of three steps:

1. Identification of a suitable model, i.e. the identification of the $p$, and $q$ order for the $\operatorname{ARMA}(p, q)$ model.

2. Estimation of the coeficients $\phi$ and $\theta$.

3. Diagnosis, i.e. the evaluation of the adequacy of the model.

\subsection{Identification}

The first step is subjective, because it is necessary to identify the underlying processes. First it must be determined the values of the integers $p$ and $q$ of the supposed $A R M A(p, q)$ process.

Parameter $d$ is the first to be achieved, because to obtain parameters $p$ and $q$ the series has to be stationary. As it can be seen in the plot of the $\mathrm{Z}$ axis sensor series (Figure 9) there is no trend and seasonally, evidencing good stationary. Thus, we are in presence of an $\operatorname{ARMA}(p, q)$ model, with zero mean.

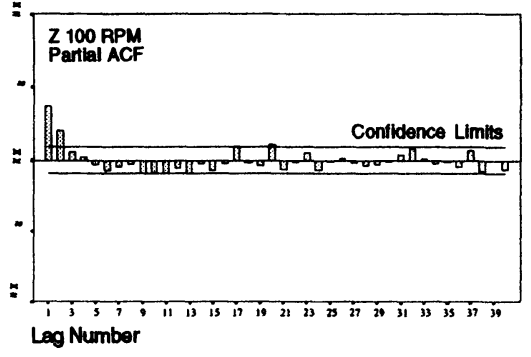

Figure 10 Plotting of PACF axis X sensor

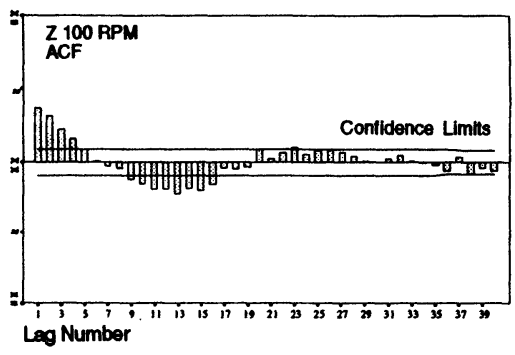

Figure 11 Plotting of ACF axis $\mathrm{Z}$ sensor data

To identify the values for $p$ and $q$, the analysis of autocorrelation function (ACF) and partial autocorrelation function (PACF), calculated at lags $1,2, \ldots$, reveals the correct values of $p$ and $q$. There is a relationship between $\operatorname{ARMA}(p, q)$ models and the corresponding data series ACF and PACF (Box.Jenkins 1970).

Analysing the plots of PACF (Figure 10) and ACF (Figure 11) for the axis $\mathrm{Z}$ sensor data, it can be concluded that we are in presence of an $\operatorname{ARMA}(2,0)$ model, with $\phi 1$ and $\phi 2>0$.

\subsection{Estimation}

After knowing the order of the model, the next step is the coeficients' estimation for an $\operatorname{ARMA}(2,0)$ model: $\phi 1$ and $\phi 2$. To perform this task, that requires a lot of computation, it was used the SPSS statistical package (SPSS 1993 ). The results obatained are presented in table 1.

Several statistics describ how well the model fits the data. The standard error, the Log likelihood, the Akaike Information Criterion (AIC) and the Schwartz Bayesian Criterion (SBC). They measure how well the model fits the series, taking into account the fact that a more elaborate 
model is expected to fit better. The AIC is for $\operatorname{ARMA}(p, 0)$ models while the SBC is a more general criterium. Using these statistics, we can choose from different models for a given serie. The model with lowest AIC, SBC, standard error and highest Log likelihood is the best.

Inspecting the estimated standard error of the coeficientes it can be verified that the coefficientes are statistically significant, and they are not correlated between them.

Table 1 Result of $\phi 1$ and $\phi 2$ estimation

\begin{tabular}{|c|c|c|c|c|c|}
\hline $\begin{array}{l}\text { Number of residuals } \\
\text { Standard error }\end{array}$ & $\begin{array}{l}512 \\
.0610640\end{array}$ & & Correlation & $\begin{array}{r}\text { Matrix: } \\
\text { AR1 }\end{array}$ & AR2 \\
\hline \multirow[t]{2}{*}{ Log likelihood } & 705.8138 & & $\phi 1$ & 1.0000000 & -.4276129 \\
\hline & & & $\phi 2$ & -.4276129 & 1.0000000 \\
\hline $\begin{array}{l}\text { Variables in the MC } \\
\text { PROB. }\end{array}$ & $\begin{array}{l}\text { del: } \\
\text { SEB }\end{array}$ & T-RATIO & APPROX. & & \\
\hline$\phi 1 \quad .32809913$ & .04311531 & 7.6098048 & .000000 & AIC & -1407.6276 \\
\hline .23221007 & .04312045 & 5.3851500 & .000000 & SBC & -1399.151 \\
\hline
\end{tabular}

Table 2 - ACF error data in low-resolution

\begin{tabular}{|c|c|c|c|c|c|c|c|c|c|c|c|c|}
\hline $\begin{array}{l}\text { Auto- } \\
\text { Lag }\end{array}$ & $\begin{array}{l}\text { Stand. } \\
\text { Corr. }\end{array}$ & Err. -1 & -.75 & -.5 & -.25 & 0 & .25 & .5 & .75 & 1 & Box-Ljung & Prob. \\
\hline $\begin{array}{l}1 \\
2 \\
3 \\
4 \\
5 \\
6 \\
7 \\
8\end{array}$ & $\begin{array}{r}.054 \\
-.018 \\
.100 \\
-.002 \\
.020 \\
.019 \\
-.009 \\
-.023\end{array}$ & $\begin{array}{l}.070 \\
.070 \\
.070 \\
.069 \\
.069 \\
.069 \\
.069 \\
.069\end{array}$ & & & . & $\begin{array}{l}\text { ! } \\
\text { ! } \\
\text { * } \\
\text { * } \\
\text { * } \\
\text { * }\end{array}$ & : & & & & $\begin{array}{r}.593 \\
.661 \\
2.729 \\
2.730 \\
2.815 \\
2.893 \\
2.908 \\
3.020\end{array}$ & $\begin{array}{l}.441 \\
.719 \\
.435 \\
.604 \\
.728 \\
.822 \\
.893 \\
.933\end{array}$ \\
\hline
\end{tabular}

\subsection{Diagnosis}

To validate the achieved model, the following checks are essential: The ACF and PACF of the error series should not be significantly different from 0 . And the residuals should be without pattern (i.e. should be white noise). A common test for this is the Box-Ljung $Q$ statistic. To accept it, this statistic should not be significant. Inspecting table 2, we found that the values of the BoxLjung statistic for the ACF function is not statisticaly significant at any lag.

\subsection{The best $A R M A(p, q)$ model for axis $\mathrm{Z}$ sensor data (100 RPM)}

Considering the analysis already performed, we can state that we are in presence of an $\operatorname{ARMA}(2,0)$ model with coeficientes $\phi 1=0.328$ and $\phi 2=0.232$.

The stochastic process $g[n]$ for axis Z sensor data (100 RPM) satisfies the following difference stochastic equation:

$g[n]-0.328 g[n-1]-0.232 g[n-2]=\varepsilon[n]$

where $\varepsilon[n]$ is a white noise process. The corresponding system transfer function is:

$H(z)=\frac{1}{1-0.328 z^{-1}-0.232 z^{-2}}$

Figure 12 depicts the axis $Z$ sensor data $(100 \mathrm{RPM})$ and the fitted $A R M A(2,0)$ model. Figure 13 shows the error data obtained from the axis $\mathrm{Z}$ sensor data (100RPM) and the fitted $\operatorname{ARMA}(2,0)$ model. As it can be seen it is white noise.

\subsection{Results obtained}

The models obtained for the current, machining sound and axes' vibrations sensors, are presented in table 3. Because of space limitations we only present the coeficients for the sound sensor in table 4 . 

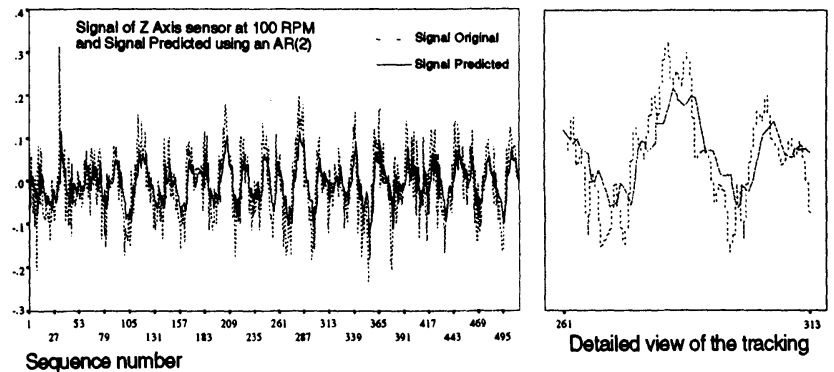

Figure 12 Axis $\mathrm{Z}$ sensor and fitted $A R M A(2,0)$ model data.
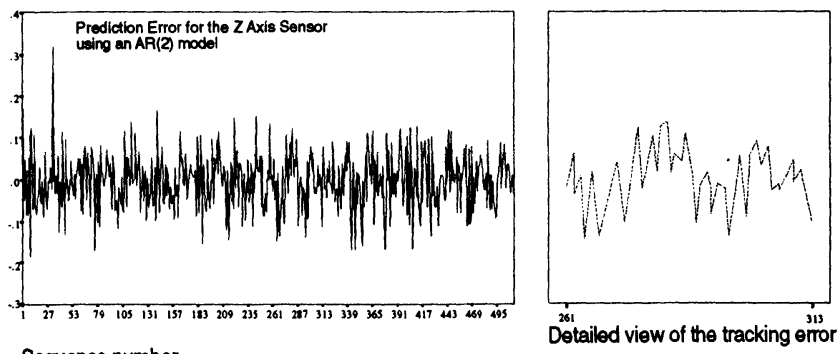

Sequence number

Figure 13 Plotting of error data.

The applicability of these models for building feature vectors as previouslly described in paragraph 5 and figure 5 a), was also confirmed experimentally. The extracted features have good clustering regions well separated, meaning their adequacy for a process status pattern recognition identifier.

Table 3 - ARIMA models achieved for diferent sensor data

\begin{tabular}{cccccccc}
\hline & \multicolumn{3}{c}{ Idle } & & Cuting & Cuting \\
ARIMA Model & $\mathbf{1 0 0} \mathbf{R P M}$ & $\mathbf{3 0 0} \mathbf{R P M}$ & $\mathbf{5 0 0} \mathbf{R P M}$ & $\mathbf{7 0 0}$ RMP & $\mathbf{9 0 0}$ RPM & 300 RPM & 500 RPM \\
\hline Current & $\operatorname{AR}(1)$ & $\operatorname{AR}(1)$ & $\operatorname{AR}(1)$ & $\operatorname{AR}(1)$ & $\operatorname{AR}(1)$ & & $\operatorname{AR}(1)$ \\
Sound & $\operatorname{AR}(4)$ & $\operatorname{AR}(3)$ & $\operatorname{AR}(3)$ & $\operatorname{AR}(3)$ & $\operatorname{AR}(3)$ & & $\operatorname{AR}(3)$ \\
Axis X & $\operatorname{ARI}(2,1)$ & $\operatorname{ARI}(2,1)$ & $\operatorname{ARI}(2,1)$ & $\operatorname{ARI}(2,1)$ & $\operatorname{ARI}(2,1)$ & $\operatorname{ARI}(3,1)$ & \\
Axis Z & $\operatorname{AR}(2)$ & $\operatorname{AR}(4)$ & $\operatorname{ARMA}(1,2)$ & $\operatorname{ARMA}(2,3)$ & $\operatorname{ARMA}(2,3)$ & $\operatorname{ARMA}(2,1)$ & \\
\hline
\end{tabular}

Table 4 - Estimated coefficientes for the machining sound model

\begin{tabular}{|c|c|c|c|c|c|}
\hline \multicolumn{5}{|c|}{ Idle } & \multirow{2}{*}{$\begin{array}{l}\text { Cuting } \\
\text { 500 RPM }\end{array}$} \\
\hline 100 RPM & 300 RPM & 500 RPM & 700 RMP & 900 RPM & \\
\hline $\begin{array}{c}\phi_{1}, \phi_{2}, \phi_{3}, \phi_{4} \\
1.20,-.37, .59,-.45\end{array}$ & $\begin{array}{c}\phi_{1}, \phi_{2}, \phi_{3} \\
.71,-.36, .60\end{array}$ & $\begin{array}{l}\phi_{1}, \phi_{2}, \phi_{3} \\
.58, .40, .72\end{array}$ & $\begin{array}{c}\phi_{1}, \phi_{2}, \phi_{3} \\
.44,-.37, .81\end{array}$ & $\begin{array}{l}\phi_{1}, \phi_{2}, \phi_{3} \\
.33,-.26, .76\end{array}$ & $\begin{array}{ccc}\phi_{1}, & \phi_{2}, & \phi_{3} \\
.85, & -.53, .49\end{array}$ \\
\hline $1.20, . .46, .67,-.43$ & $.80,-.39, .53$ & $.58, .39, .71$ & $.39,-.27, .74$ & $.37,-27, .76$ & $.99,-.64, .45$ \\
\hline $1.19,-.35, .50,-.40$ & $.81,-.45, .60$ & $.57, .45, .74$ & $.36,-.31, .81$ & $.36,-.27, .74$ & $.72,-.40, .61$ \\
\hline
\end{tabular}




\section{CONCLUSIONS AND FUTURE WORK}

A methodology for characterising the machining process is proposed. It is based on sensorial data modelling using autoregressive $\operatorname{ARMA}(p, q)$ models for stochastic signal characterisation. The relationship between the $\operatorname{ARMA}(p, q)$ models obtained and system transfer function theory is presented to explain the meaning of the models obtained and their relationship with the real system under observation. Because of the complexity of the machining process, it is also proposed a method for modelling the complete process as a sequence of elementary and more simple subprocesses. Each one of these simple sub-process corresponds to the NC instructions under execution.

The applicability of the proposed methodology was assessed by doing preliminary experiments on a laboratorial lathe. The results obtained, are very encouraging for pursuit the proposed approach in future works.

Future work will be done in two directions: first, pursuit the research for characterisation of more situations of the machining process and second, develop real-time monitors based on our approach. The integration of the proposed approach in our Prognostic and Monitoring System for CNC Machines, (for detailed description see (Barata 94)) will be done. Tansputer tasks will carry out all the real time processing necessary for doing the estimation of the $\operatorname{ARMA}(p, q)$ parameters. The applicability of adaptive systems described in (Widrow 1985) seems to be an interesting approach for real time parameters estimation.

\section{REFERENCES}

Abraham, Bovas and Johannes Ledolter(1983). Statistical Methods for Forecasting. Jhon Wiley \& Sons, New York, 1983.

Barata, Manuel M. (1990a). Maintenance and Prognostic Systems: State of the Art. Internal report UNL DI NT-2-90 GR RP-PS-90.

Barata, Manuel M., José C. Cunha and A. Steiger-Garção (1990b). Transputer Environment to Support Heterogeneous Systems in Robotics. Transputer Applications 90 Conference, Southampton, 11-13 July, 1990.

Barata, Manuel M. and A. Steiger-Garção (1990c). Sensor Environment for Prognosis and Monitoring Systems Support. 3rd ISRAM International Symposium on Robotics and Manufacturing, British Columbia, Canada, 20-22 July, 1990.

Barata, Manuel M., Thomas W. Rauber, A. Steiger-Garção (1992). Sensor Integration for Expert CNC Machines Supervision. Etfa'92 Emerging Technologies in Factory Automation. Melbourne, Autralia, 11-14 August, 1992.

Barata, Manuel M., Thomas W. Rauber, A. Steiger-Garção (1994). Prognostic and Monitoring System for CNC machines. Revue Européenne Diagnostic et Sûreté de Fonctionnement. Editions HERMES, Vol. 4 - № 2/1994, 1994.

Box- Jenkins, George E. P. and Gwilyn M. Jenkins (1970). Time Series Analysis Forecasting and control. Holden-Day, San Francisco, 1970.

Steiger-Garção, A., M. M. Barata and L. F. S. Gomes (1989). "Integrated Environment for Prognosis and Monitoring System Support". Ist UNIDO workshop on Robotics and Computer Integrated Manufacturing. Lisbon, Portugal, 11-15 September, 1989.

Guinea, D. et al.(1991). Multi-sensor integration - An automatic feature selection and state identification methodology for tool wear estimation. Computers In Industry, Vol. 17 , no. 2\&3, pp 121-130, Nov. 91 . 
Harvey, Andrew C. (1992). Forecasting, Structural Time Series and the Kalman Filter. Cambridge University Press, 1982.

Papoulis, Athanasios (1965). Probability, Random Variables and Stochastic Processes. McGrawHill International Students Editions, 1965.

Papoulis, Athanasios (1984). Signal Analysis. McGraw-Hill International Editions, 1982.

Rauber, Thomas W., Manuel M. Barata e A. Steiger-Garção (1993). A Toolbox for Analysis and Visualization of Sensor Data in Supervision. TOOLDIAG'93, Toulouse-France, April 5-7, 1993.

SPSS, Inc. (1993), SPSS Statistical Package for Windows Release 6.0. SPSS Inc., $444 \mathrm{~N}$. Michigan Avenue, Chicago, Illinois 60611, 1993.

Widrow, B. and S.D.Stearns (1985). Adaptive signal Processing. Prentice-Hall, Englewood cliffs, N.J., 1985.

\section{BIOGRAPHY}

Manuel M. Barata is adjunct professor at Instituto Superior de Engenharia de Lisboa of the Polytechnic Institute of Lisbon where he teaches computer science subjects. Since 1987 he has joined the Intelligent Robotics Centre of the UNINOVA Institute, where he is finishing his Ph.D. thesis on Monitoring and Prognostic Systems for CNC Machines. His main research interests are: Monitoring Systems, Real-time architectures, Transputer based systems and Artificial Intelligence systems for real-time operation. He has participated in several international European projects and has been the group leader of the UNINOVA participation in the CIMTOFI BRITE/EURAM project.

Adolfo Steiger-Garção is full professor at Department of Electrical Engineering of the New University of Lisbon. He is also the president of UNINOVA Institute and the director of the Intelligent Robotics Centre. His main research interests are: Robotics / Perception / Monitoring. $\mathrm{He}$ is responsible for several international projects and has more than 100 publications on national and international conferences.

Ricardo R. Jardim Gonçalves is graduated in Computer Science, and now is coursing his master in Operational Research and Systems Engineering. He has a large experience in the areas of computer science research specially in integration of industrial tools and standards, STEP in particular. He his the working group leader of the UNINOVA participation in the ESPRIT III RoadRobot project and has participated has work coordinator in the CIMTOFI BRITE/EURAM project .

José Álvaro Assis Lopes is associated professor at the Autonomous Section of Economy and Management in the Instituto Superior Técnico of Technical University of Lisbon. He has been graduated in Chemical Engineering, got a master degree on Operational Research, and made a Ph.D. on Operational Research and Systems Engineering. His main research interests are: Forecasting, Operational Research and Management Science. 